\title{
An elevated D-dimer value: a burden on our patients and hospitals
}

This article was published in the following Dove Press journal:

International Journal of General Medicine

24 January 2012

Number of times this article has been viewed

\author{
Nitin Chopra \\ Pradeep Doddamreddy \\ Hermanjeet Grewal \\ Pratap C Kumar \\ Jackson Park Hospital, Chicago, \\ IL, USA
}

Correspondence: Nitin Chopra

Jackson Park Hospital 753 I S. Stony Island

Ave, Rm\#I72 Chicago IL, 60649, USA

Phone +I 3129521082

Fax + I 6307890394

Email nitinchopra I I@hotmail.com
Abstract: With 200,000 annual deaths in the United States due to pulmonary embolism (PE), efficient and accurate diagnosis is mandatory. Since negative D-dimer values are only useful in ruling out PE, elevated values alone should not result in excessive testing. This study assessed the diagnostic and financial yield of the D-dimer in diagnosing PE. This retrospective review of 220 medical records of patients at a South Chicago Community Hospital explored the extent of the work-up following an elevated D-dimer for a suspected PE. Patients were randomly selected with no exclusion criteria. Five of the 118 (4.2\%) patients with elevated D-dimer values were diagnosed with a PE. Tests ordered based on elevated D-dimer values were billed for more than $\$ 200,000$. The current diagnostic approach has been medically and financially inefficient. Patients should not be worked-up for a PE based primarily on an elevated D-dimer value. Two prominent factors, independent of $\mathrm{PE}$, that result in elevated $\mathrm{D}$-dimer values and were pertinent to the studied population, are age and African-American origin. Implementing a scoring system, like the revised-Geneva scale, will establish a better index of suspicion to improve both the physician's diagnostic approach and the yield of the work-up.

Keywords: pulmonary embolism, D-dimer, diagnosis, age, African-Americans, scoring

\section{Practice recommendations}

This evaluation illustrated that without the implementation of an appropriate scaling system, the work-up to rule out PE is of low yield. Consequences of excessive testing include unnecessary exposure to radiation and financial wastefulness.

The implementation of clinical prediction rules will improve the diagnostic approach, management, and utilization of resources.

The use of a scaling system will also account for the possibility of normal physiologic or pathologic conditions, other than $\mathrm{PE}$, that may attribute to the elevation of D-dimer values.

\section{Introduction}

Pulmonary Embolism (PE) is a very dangerous disease state, as it presents with nonspecific symptoms that can lead to death within a few hours of onset. As many as 200,000 deaths per year in the United States are due to PE, making this the third leading cardiovascular cause of death of inpatient groups. ${ }^{1}$ Any patient who is at risk for venous thrombosis must be watched carefully, since $50 \%$ to $60 \%$ of these patients can develop PE. ${ }^{1}$ There has been a corresponding rise in diagnostic studies being conducted based on suspicion of PE. 
The rates of unexpected death due to $\mathrm{PE}$ are second only to cardiac arrest. Of those patients who develop PE, approximately $10 \%$ will die within the first hour, while another $30 \%$ will die from a recurrent case of emboli. ${ }^{2}$ With improved and increased accuracy of diagnosing PE, $25 \%$ of those who currently die from PE could be saved. ${ }^{2}$ However, the concern of missing the diagnosis of PE should not give way to unnecessary orders for tests without adequate basis for those orders.

It has been noted in the past that the D-dimer value can be systematically elevated in cases of deep venous thrombosis or PE. However, the D-dimer value can be elevated in a number of other normal physiologic, as well as pathologic states. Hence finding the D-dimer level to be elevated should not be the sole basis for a PE workup. Rather, clinical suspicion should direct the investigation for the presence of PE. With the aid of appropriate labs and imaging studies, the diagnosis of PE can be made quickly and adequate treatment can be initiated in a short period of time. The purpose of this study is to demonstrate that an elevated D-dimer value alone often results in extensive PE workup, which has a low diagnostic and economical yield.

\section{Method}

A retrospective analysis of the diagnosis of PE at Jackson Park Hospital (JPH) was conducted. JPH is a short-term, comprehensive care facility serving the south side of Chicago, IL. The hospital serves a very high indigent, adult, African-American population. The analysis focused on the use of the D-dimer and CT scan of the thorax with contrast. In an attempt to minimize bias, there were no exclusion criteria. The charts of 220 patients having a D-dimer test ordered at the hospital were randomly selected for review. Factors evaluated for these patients included levels of the HemosIL assay of the D-dimer and subsequent tests ordered, including ventilation/perfusion scan (V/Q scan), Doppler studies and CT scan of the thorax with contrast.

To assess the consistency in the approach to diagnosing a PE, the sequence of events leading to the suspicion and ultimate diagnosis was considered in the patients diagnosed with a PE. The factors for each of the cases that were considered included clinical suspicion, D-dimer, the use of arterial blood gases, V/Q scan, Doppler studies, and CT of the thorax with contrast. While it was possible to investigate these tests, the current lack of a widely accepted and used PE protocol at the hospital resulted in limitations to objectively identifying the ordering physician's clinical and laboratory rationale behind the tests.

\section{Results}

The study consisted of reviewing 220 patients who had one or more D-dimer tests ordered. Of the 220 patients, 217 patients had D-dimer values. The three patients without values had the test done but results were not available. 118 out of the 217 patients $(54.4 \%)$ had elevated D-dimer values greater than $254 \mathrm{ng} / \mathrm{mL}$. Of these 118 patients, five were diagnosed with pulmonary emboli, resulting in a positive predictive value of $4.2 \%$.

Ninety-two follow up tests were ordered for the 118 patients with elevated D-dimer values. Some of these patients may have had more than one follow-up test ordered while some patients had no further tests ordered. Thirty-four of the 92 tests ordered were venous Doppler studies (36.9\%), 36 were CT of the thorax (39.1\%) and 22 were V/Q scans (23.9\%). Out of the 118 patients with elevated D-dimer, five were diagnosed with $\mathrm{PE}$.

\section{Cost analysis}

When considering the group of patients who had the D-dimer test ordered, those having elevated values often had subsequent tests ordered, including V/Q scans, CT scans of the thorax with contrast and lower venous Doppler studies. Summarized in the Table 3 is average cost and reimbursement (as per Medicaid) for the tests ordered in the 118 cases of elevated D-dimer values.

\section{Discussion}

The prevalence of PE and the associated mortality undoubtedly warrant sufficient work-up when there is clinical suspicion. The current approach to diagnosing PE has been inefficient, as there is an overly liberal use of the D-dimer lab test and chest CT scan. This exposes the patient to high doses of radiation, increases costs, and delays the disposition of patients. ${ }^{3}$ It is evident that the diagnostic approach must be altered to efficiently and effectively diagnose pulmonary emboli.

The D-dimer lab test is considered to be highly sensitive, but non-specific. ${ }^{4}$ As a result, the role of this test has typically been limited to ruling out a PE in cases of low suspicion. ${ }^{4}$ However, there are various formulations of the D-dimer test itself, each with different sensitivities. The HemosIL assay, used at JPH, has sensitivities comparable to the gold standard VIDAS ELISA assay. ${ }^{5}$ Due to such high sensitivity, when a D-dimer assay result is negative, PE can be confidently ruled out. Sensitivity, negative predictive value, turnaround time for the results and cost all play important roles when selecting a particular D-dimer assay. Turnaround time is important since a PE needs to be ruled out as quickly as possible. 
Cost-effective assays are important in community hospitals like JPH. Although the ELISA may be the gold standard assay, since the sensitivity, negative predictive value, turnaround time, and cost of the new generation latex agglutination D-dimer assays are comparable and in some instances better, they are more advantageous than the ELISA.

Heterogeneity is a major problem with D-dimer measurement. There has been great confusion resulting from multiple commercial assays having various techniques, cut-offs, systems of units, operational characteristics, and clinical validity. Since D-dimer is a complex mixture of degradation products of different sizes, standardization has not been possible. ${ }^{6}$

While many assays typically use cut-off values of $500 \mathrm{ng} / \mathrm{mL}$, studies found that the lower cut-off values with the HemosIL assay demonstrated not only safe exclusion of venous thromboembolism, but also proved specificity and exclusion rates similar to other formulations. ${ }^{5}$ As a result, at $\mathrm{JPH}$, the threshold for the D-dimer value is $254 \mathrm{ng} / \mathrm{mL}$. However, according to the literature, D-dimer values greater than $500 \mathrm{ng} / \mathrm{mL}$ are considered positive. ${ }^{7}$ If the threshold for an elevated D-dimer had been increased to $500 \mathrm{ng} / \mathrm{mL}$ in this study of the 217 patients from the D-dimer group, 66 would have had an elevated D-dimer, of whom 5 were diagnosed with a PE. A distribution of these D-dimer values is illustrated in Figure 1. Although a normal D-dimer value is used to rule out PE, an elevated D-dimer value alone cannot indicate a PE. There are many factors that can contribute to the elevation of a D-dimer lab value. D-dimer can be elevated in advanced age, pregnancy, trauma, post-operative periods, inflammatory states, and cancer ${ }^{4}$ (Table 1). Also, it has been found that the D-dimer values are markedly higher in African-Americans, who are four times more likely to have an extreme value $(>600 \mathrm{ng} / \mathrm{mL})$ than Caucasians. ${ }^{8}$ This is significant because the vast majority of the patient population at JPH is of African-American origin.

In a study by Harper et $\mathrm{al},{ }^{9}$ it has been found that the median D-dimer concentration increased with age from $294 \mathrm{ng} / \mathrm{mL}$ in people aged $16-40$ years; to $387 \mathrm{ng} / \mathrm{mL}$ in those aged $40-60$ years; to $854 \mathrm{ng} / \mathrm{mL}$ in those aged $60-80$ years; to $1397 \mathrm{ng} / \mathrm{mL}$ in those aged $80+$ years. The assay specificity decreased with age from $70 \%$ in patients $<40$ years to less than $5 \%$ in patients $>80$ years. ${ }^{9}$ This trend is of particular interest because age is a risk factor for PE as well as contributing to an elevated D-dimer. The D-dimer values of the 220 patients in different age groups, both in the literature ${ }^{8}$ and at $\mathrm{JPH}$, is presented in Table 2. While the average value is increased in the older groups, it is possible that in a larger follow-up analysis, the trend may correlate more with that seen in the study conducted by Harper et al. ${ }^{9}$ Douma et $\mathrm{al}^{10}$ found that an age-adjusted D-dimer cut-off point, combined with clinical probability, generally increased the proportion of older patients in whom PE could be safely excluded. Raising the cut-off value of the D-dimer test for older patients to values between $600 \mathrm{ng} / \mathrm{mL}$ and $1000 \mathrm{ng} / \mathrm{mL}$ increases the test's specificity, but at the cost of safety. They suggested a new D-dimer cut-off value to be (patient's age $\times 10) \mu \mathrm{g} / \mathrm{L}$ in patients older than fifty years. ${ }^{10}$

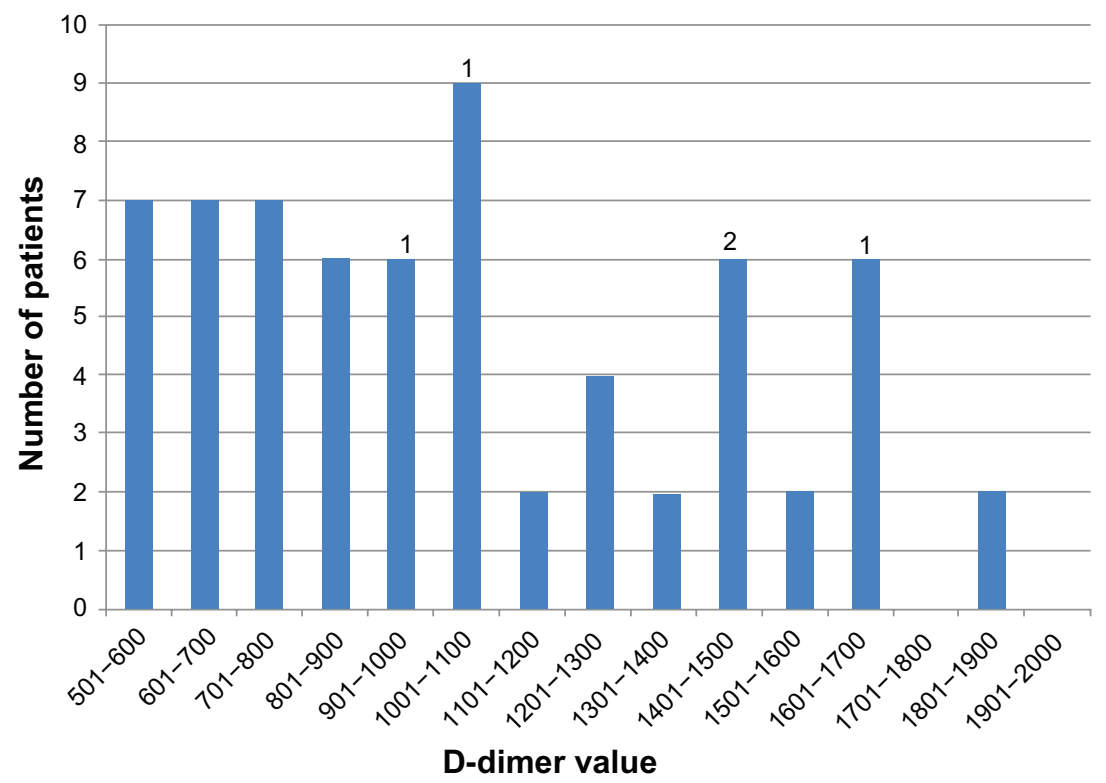

Figure I A distribution of the 66 out of 217 D-dimer values that were above $500 \mathrm{ng} / \mathrm{mL}$. Note: The 5 diagnosed pulmonary embolisms are represented numerically in the corresponding D-dimer value. 
Table I Disorders associated with an increased D-dimer value

Arterial thromboembolic disease

Nephrotic syndrome (eg, renal vein thrombosis)

Normal pregnancy

Severe infection/sepsis/inflammation

Surgery/trauma (eg, tissue ischemia, necrosis)

Intracardiac thrombus

Venous thromboembolic disease

Acute limb ischemia

Pulmonary embolism

Vasoocclusive episode of sickle cell disease

Preeclampsia and eclampsia

Abnormal fibrinolysis; use of thrombolytic agents

Chronic renal failure and underlying cardiovascular disease

Malignancy

Cardiovascular disease, congestive failure

Deep vein thrombosis

Atrial fibrillation

Acute renal failure

Renal disease

Systemic inflammatory response syndrome

Severe liver disease (decreased clearance)

Myocardial infarction

Stroke

Disseminated intravascular coagulation

Of the five patients diagnosed with a PE, four were older than 60 years. The diagnostic value of the D-dimer must thus be cautiously considered. More than $95 \%$ of the 220 patients investigated were African American. Of the patients studied, $17.1 \%$ were between the ages of $16-39$ years, $46.1 \%$ were between the ages of $40-59,28.6 \%$ were between the ages of $60-79$, and $7.8 \%$ were over 80 years of age. The literature indicates that the positive predictive value of the D-dimer test in diagnosing a PE is approximately $20 \% .{ }^{11}$ At JPH the PPV of $4.2 \%$ was markedly less. This may be due to the elevated D-dimer values in the predominantly African American and elderly population at the hospital.

The combination of the present clinical judgment and rationale by different physicians in the evaluation of $\mathrm{PE}$ has resulted in very low yields from the tests. The common practice has been either over-defensive, neglected to account for other possible causes of elevated D-dimer values, or a combination of both. This disparity further supports the need

Table 2 A comparison between the literature and JPH of the D-dimer values $(\mathrm{ng} / \mathrm{mL})$ for each age group

\begin{tabular}{lll}
\hline Age group & $\begin{array}{l}\text { Literature median } \\
\text { D-dimer }\end{array}$ & $\begin{array}{l}\text { Mean D-dimer } \\
\text { in JPH study }\end{array}$ \\
\hline $16-39$ & 294 & 490 \\
$40-59$ & 387 & 400 \\
$60-79$ & 854 & 552 \\
$80+$ & 1397 & 899 \\
\hline
\end{tabular}

Abbreviation: JPH, Jackson Park Hospital (Chicago, IL). to consider normal physiologic and pathologic conditions, other than PE, that can cause an elevated D-dimer. Moderate sensitivity and specificity of clinical signs and symptoms of PE prevent the diagnosis from being made solely on a clinical basis. The traditional gold standard of pulmonary angiography is invasive and resource demanding. Submitting all patients suspected of having a PE to imaging would not be cost effective. To efficiently and cost-effectively work-up a suspected PE, institution-wide clinical prediction rules must be implemented. ${ }^{12}$

Due to the high false positive rate of the D-dimer, there has been an increase in the use of radiological imaging, length of patient stay, and false positive diagnoses. ${ }^{13}$ The diagnosis of any illness always begins with a thorough patient history and physical exam. The first step for the diagnosis of a PE should also be the same. The most extensively validated guidelines have been the Wells and Geneva scores. These initial guidelines require diagnostic tests, such as chest x-ray or arterial blood gas analysis on room air, that are not always available. More recent rules, like the revised-Geneva scoring scale, are based only on clinical elements and it has been demonstrated that patients can be safely managed based on clinical assessment utilizing these scores. Implementing a scoring system (Figure 2) would establish an appropriate index of suspicion and improve the diagnostic approach towards PE. ${ }^{14}$ It would also reduce the financial burden of over-testing for suspected PE by increasing the yield of the work-up.

The most significant change in the diagnostic approach to acute and chronic PE during the past years has been the utilization of the spiral CT scan. Many clinicians have used the spiral CT scan in situations when other diagnostic techniques, for example the ventilation/perfusion scan, have failed to yield a definite diagnosis. According to a study by Ost et al, ${ }^{15}$ the spiral CT has led to an improvement in the diagnosis rate of PE from 1.8 per 1000 hospital admissions to 2.8 per 1000 admissions. In addition to this diagnostic improvement, the perception of the spiral CT being a noninvasive technique has made it favourable. Even with the diagnostic improvement of the spiral CT, only one third of patients suspected of PE actually were diagnosed with a PE in the study by Ost et al. The remaining two thirds often had such conditions as aortic dissection, pneumonia, lung cancer, metastasis and pneumothorax, all of which are conditions that could be diagnosed with the aid of the CT scan. Despite this advancement, there are numerous inconclusive CT scan results. ${ }^{15}$ These results may be attributed to the fact that current techniques for CT arteriography have the greatest 


\begin{tabular}{|c|c|c|c|c|c|}
\hline \multicolumn{2}{|l|}{ Wells score } & \multicolumn{2}{|l|}{ Geneva score } & \multicolumn{2}{|l|}{ Revised Geneva score } \\
\hline Items & Score & Items & Score & Items & Score \\
\hline Previous PE or DVT & 1.5 & Previous PE or DVT & 2 & Age $>65$ years & 1 \\
\hline Heart rate $>100$ & 1.5 & Heart rate $>100$ & 1 & Previous DVT or PE & 3 \\
\hline Recent surgery or immobilization & 1.5 & Recent surgery & 3 & Surgery or fracture within 1 month & 2 \\
\hline Clinical signs of DVT & 3 & Age & & Active malignancy & 2 \\
\hline Alternative diagnosis less likely than PE & 3 & $60-79$ & 1 & Unilateral lower limb pain & 3 \\
\hline Hemoptysis & 1 & $\geq 80$ & 2 & Hemoptysis & 2 \\
\hline \multirow{12}{*}{ Cancer } & 1 & Arterial blood gases & & Heart rate & \\
\hline & & $\mathrm{CO}_{2}(\mathrm{kPa})$ & & $75-94$ & 3 \\
\hline & & $<4.8$ & 2 & $\geq 95$ & 5 \\
\hline & & $4.8-5.19$ & 1 & $\begin{array}{l}\text { Pain on lower limb deep vein palpation } \\
\text { and unilateral edema }\end{array}$ & 4 \\
\hline & & $\mathrm{O}_{2}(\mathrm{kPa})$ & & & \\
\hline & & $<6.5$ & 4 & & \\
\hline & & $6.5-7.99$ & 3 & & \\
\hline & & $8-9.49$ & 2 & & \\
\hline & & 9.5-10.99 & 1 & & \\
\hline & & Chest X-ray & & & \\
\hline & & Atelectasis & 1 & & \\
\hline & & Elevated hemidiaphragm & 1 & & \\
\hline Clinical probability & & Clinical probability & & Clinical probability & \\
\hline Low & $<2$ & Low & $0-4$ & Low & $0-3$ \\
\hline Intermediate & $2-6$ & Intermediate & $2-6$ & Intermediate & $4-10$ \\
\hline High & $>6$ & High & $\geq 9$ & High & $\geq 11$ \\
\hline \multicolumn{6}{|l|}{ Dichotomized } \\
\hline PE unlikely & $\leq 4$ & & & & \\
\hline PE likely & $>4$ & & & & \\
\hline
\end{tabular}

Figure 2 The Wells, Geneva, and revised-Geneva pre-test possibility scores. ${ }^{6}$

Abbreviations: PE, pulmonary embolism; DVT, deep vein thrombosis.

sensitivity and specificity for detecting emboli in the main and lobar arteries. ${ }^{16}$ The spiral CT scan has a sensitivity of $57 \%-100 \%$ and specificity of $78 \%-100 \%$, both of which vary with the location of the emboli. Both values are approximately $90 \%$ in the main and lobar pulmonary arteries however there is a marked decrease in the segmental and sub-segmental pulmonary vessels. ${ }^{4}$ Even though CT scans are of great advantage, they expose the patient to substantial amounts of radiation which can lead to health risks associated with radiation exposure. Although CT scans can be useful in diagnosis of PE, an increased D-dimer should not be the sole reason for a CT scan to be ordered. The average annual natural background radiation exposure is $2.5 \mathrm{mSv}$ compared to $7.0 \mathrm{mSv}$ of radiation exposure with one CT scan.

Table 3 A breakdown of the average cost and reimbursement (by Medicaid and Medicare) of the tests commonly ordered while considering a pulmonary embolism

\begin{tabular}{|c|c|c|c|c|c|c|c|}
\hline Test & Number & $\begin{array}{l}\text { Average } \\
\text { cost }(\$)\end{array}$ & $\begin{array}{l}\text { Total cost } \\
(\$)\end{array}$ & $\begin{array}{l}\text { Average medicaid } \\
\text { reimbursement (\$) }\end{array}$ & $\begin{array}{l}\text { Total medicaid } \\
\text { reimbursement }(\$)\end{array}$ & $\begin{array}{l}\text { Average medicare } \\
\text { reimbursement (\$) }\end{array}$ & $\begin{array}{l}\text { Total medicare } \\
\text { reimbursement (\$) }\end{array}$ \\
\hline $\begin{array}{l}\text { D-dimer } \\
\text { (85379) }\end{array}$ & 118 & $\$ 256.00$ & $\$ 30,280.00$ & $\$ 7.50$ & $\$ 885.00$ & $\$ 21.24$ & $\$ 2,506.32$ \\
\hline $\begin{array}{l}\text { V/Q scan } \\
\text { (78588) }\end{array}$ & 22 & $\$ 2,400.00$ & $\$ 52,800.00$ & $\$ 65.35$ & $\$ 1,437.70$ & $\$ 374.81$ & $\$ 8,245.82$ \\
\hline $\begin{array}{l}\text { CT thorax } \\
\text { w/contrast } \\
(7 / 260)\end{array}$ & 36 & $\$ 2,800.00$ & $\$ 100,800.00$ & $\$ 75.90$ & $\$ 2,732.40$ & $\$ 4 \mid 4.97$ & $\$ 14,938.92$ \\
\hline $\begin{array}{l}\text { Lower } \\
\text { venous } \\
\text { Doppler } \\
(93970)\end{array}$ & 34 & $\$ 700.00$ & $\$ 23,800.00$ & $\$ 87.55$ & $\$ 2,976.70$ & $\$ 232.19$ & $\$ 7,894.46$ \\
\hline & & & $\$ 207,608.00$ & & $\$ 8,03 I .80$ & & $\$ 33,585.52$ \\
\hline
\end{tabular}

Notes: There were 118 patients (of the 220 total patients with the D-dimer ordered) who had an elevated D-dimer value ( $>254 \mathrm{ng} / \mathrm{mL}$ ). In parenthesis, beside the test, is the corresponding CPT code. Based on these values, the hospital is typically reimbursed between $4 \%$ and $16 \%$ of the cost of these tests.

Abbreviations: V/Q scan, ventilation/perfusion scan; CT, computerized tomography. 
This is equivalent to about 140 chest radiographs. ${ }^{17}$ Patients can experience a lifelong impact with the increased risk of developing cancer due to the cumulative effects of radiation exposure, whether from a chest radiograph, CT scan or environmental. Proper rationale should be exercised before ordering a CT scan.

\section{Conclusion}

The current approach to diagnosing pulmonary emboli must be improved. The current approach has had low yields, has been financially wasteful and has caused unnecessary radiation exposure to patients. It is imperative that patients should not be worked-up for a PE based primarily on an elevated D-dimer value, as there are numerous conditions and pre-disposing factors, other than a PE, that can cause an elevated D-dimer value. Two very prominent factors that pertained to the population examined included age and African-American origin.

It is proposed that implementing a scoring system, like the revised-Geneva scale, will establish a better index of suspicion that will ultimately guide the medical team to improve their diagnostic approach, management, and utilization of resources. Such a scale will enable patients with a low risk for $\mathrm{PE}$ to have a D-dimer ordered with the objective of ruling out a PE. Using similar scales, patients who are suspected to be highly likely to have a PE may have a CT scan of the thorax done. Such a system would be systematic and reflect sound scientific practice that would be beneficial to the patient and hospital, alike.

\section{Disclosure}

The authors report no conflicts of interest in this work.

\section{References}

1. McPhee SJ, Papadakis MA, editors. Current Medical Diagnosis and Treatment, Forty-Sixth Edition. New York: McGraw-Hill Companies Inc; 2007.

2. Sharma S. Pulmonary Embolism. 2006. Available from: http://www. emedicine.com/med/TOPIC1958.HTM.
3. Dunn KL, Wolf JP, Dorfman DM, Fitzpatrick P, Baker JL, Goldhaber SZ. Normal D-dimer levels in emergency department patients suspected of acute pulmonary embolism. J Am Coll Cardiol. 2002;40(8):1475-1478.

4. Fedullo PF, Tapson VF. Clinical Practice. The evaluation of suspected pulmonary embolism. N Engl J Med. 2003;349(13):1247-1256.

5. Arnout J, Sales M, Arza B, Sánchez T, Serra J, Moerloose P. Clinical management study of venous thromboembolism (VTE) using HemosIL ${ }^{\mathrm{TM}}$ $D$-dimer. Presented at the XX Congress of The International Society on Thrombosis and Haemostasis, August 6-12, 2005, Sydney, Australia.

6. Righini M, Perrier A, De Moerloose P, Bounameaux H. D-dimer for venous thromboembolism diagnosis: twenty years after. $J$ Thromb Haemost. 2008;6(7):1059-1071.

7. Ouellette DR. Pulmonary Embolism. 2008. Available from: http://www. emedicine.com/emerg/topic490.htm.

8. Pieper CF, Murali K, Rao K, Currie MS, Harris TB, Cohen HJ. Age, functional status, and racial differences in plasma D-dimer levels in community-dwelling elderly persons. J Gerontol A Biol Sci Med Sci. 2000;55(11):649-657.

9. Harper PL, Theakston E, Ahmed J, Ockelford P. (2006). D-dimer concentration increases with age reducing the clinical value of the D-dimer assay in the elderly. Intern Med J. 2007;37(9):607-613.

10. Douma RA, Le Gal G, Sohne M, et al. Potential of an age-adjusted D-dimer cut-off value to improve the exclusion of pulmonary embolism in older patients: a retrospective analysis of three large cohorts. $B M J$. 2010;340:c1475. (doi:10.1136/bmj.c1475).

11. Hayang JE, Manchanda PP. Predictive value of the rapid whole blood agglutination D-dimer Assay (AGEN SimpliRED) in community outpatients with suspected deep venous thrombosis. Perm J. 2006;10(1): 16-20.

12. Bounameaux H. Contemporary management of pulmonary embolism: the answer to ten questions. J Intern Med. 2010;268:218-231.

13. Kline JA, Wells PS. Methodology for a rapid protocol to rule out pulmonary embolism in the emergency department. Ann Emerg Med. 2003;42(2):266-275.

14. Ceriani E, Combescure C, Le Gal G, et al. Clinical prediction rules for pulmonary embolism: a systematic review and meta-analysis. JThromb Haemost. 2010;8(5):957-970.

15. Ost D, Khanna D, Shah R, et al. Impact of spiral computed tomography on the diagnosis of pulmonary embolism in a community hospital setting. Respiration. 2004;71(5):450-457.

16. Tapson VF. Acute pulmonary embolism. N Engl J Med. 2008;358(10): 1037-1052.

17. Mayo J, Aldrich J, Muller N. Radiation exposure at chest CT: a statement of the Fleischner Society. Radiology. 2003;228(1):15-21.
International Journal of General Medicine

\section{Publish your work in this journal}

The International Journal of General Medicine is an international, peer-reviewed open-access journal that focuses on general and internal medicine, pathogenesis, epidemiology, diagnosis, monitoring and treatment protocols. The journal is characterized by the rapid reporting of reviews, original research and clinical studies across all disease areas.

\section{Dovepress}

A key focus is the elucidation of disease processes and management protocols resulting in improved outcomes for the patient. The manuscript management system is completely online and includes a very quick and fair peer-review system. Visit http://www.dovepress.com/ testimonials.php to read real quotes from published authors. 